\title{
Publisher Correction: A silicon singlet-triplet qubit driven by spin-valley coupling
}

Ryan M. Jock (D), N. Tobias Jacobson, Martin Rudolph, Daniel R. Ward(D, Malcolm S. Carroll \& Dwight R. Luhman

Correction to: Nature Communications https://doi.org/10.1038/s41467-022-28302-y, published online 02 February 2022.

The original version of this Article contained an error in Fig. 3, in which the blue and green vertical dashed lines were missing in panel f. The correct version of Fig. 3 is:

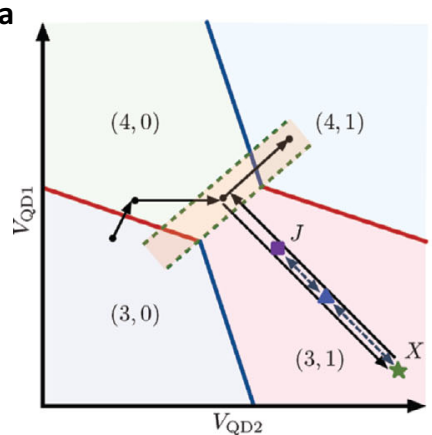

b
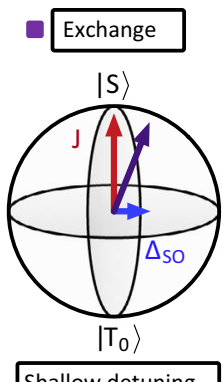

Shallow detuning

Singlet Return (arb. units)

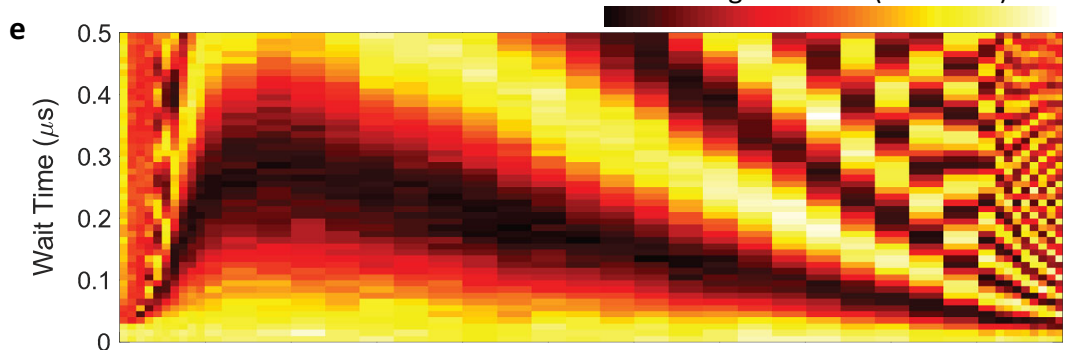

f

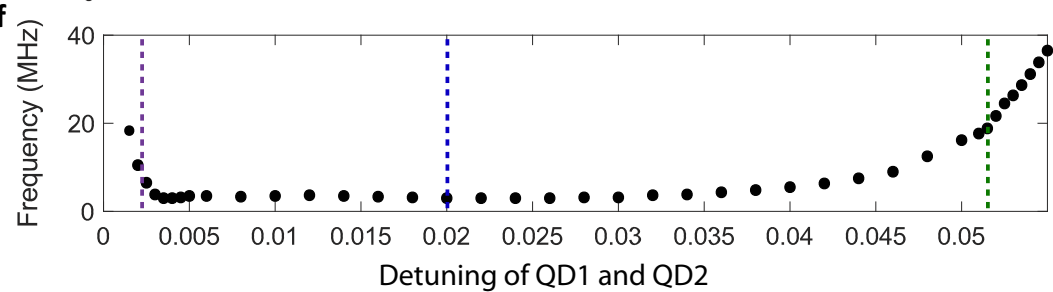

d $\quad+$ Hot spot
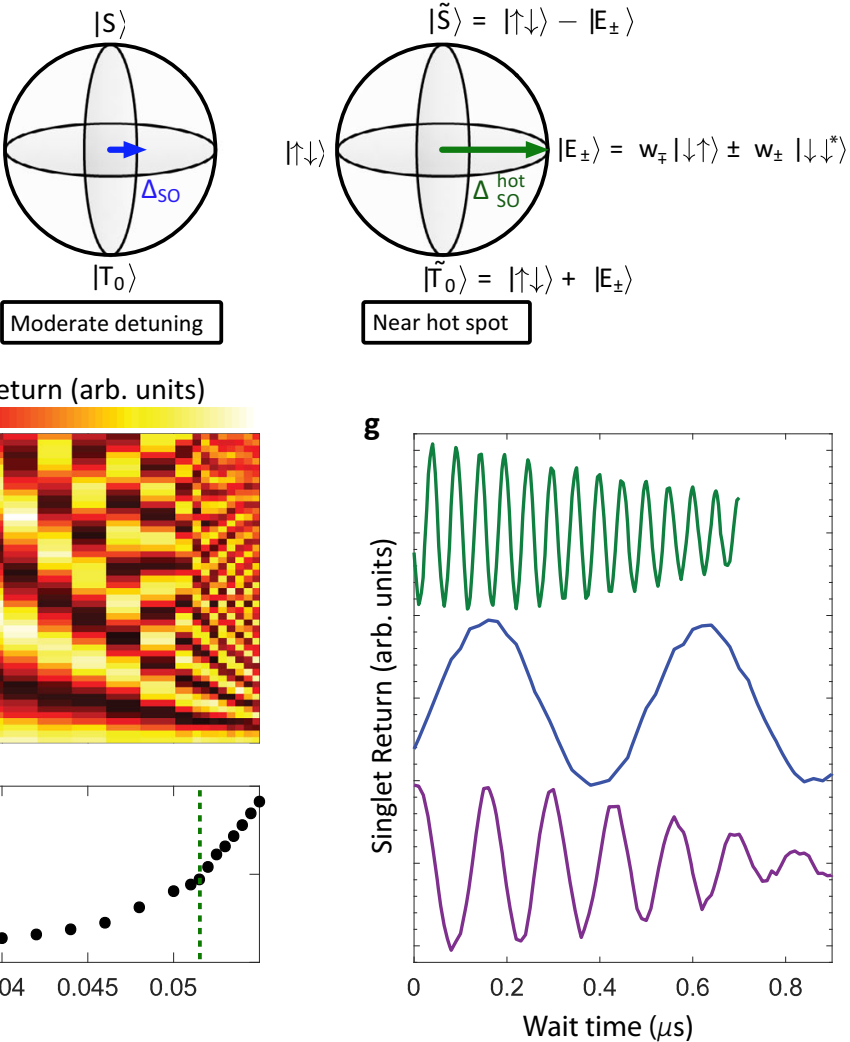
which replaces the previous incorrect version:

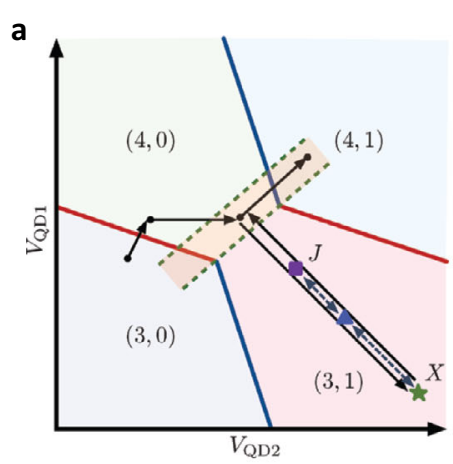

b

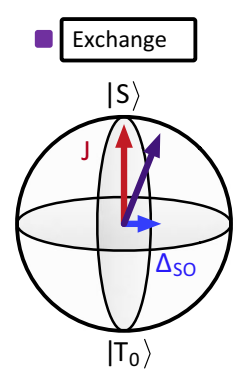

Shallow detuning
C
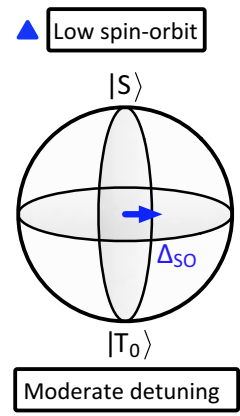

Singlet Return (arb. units)

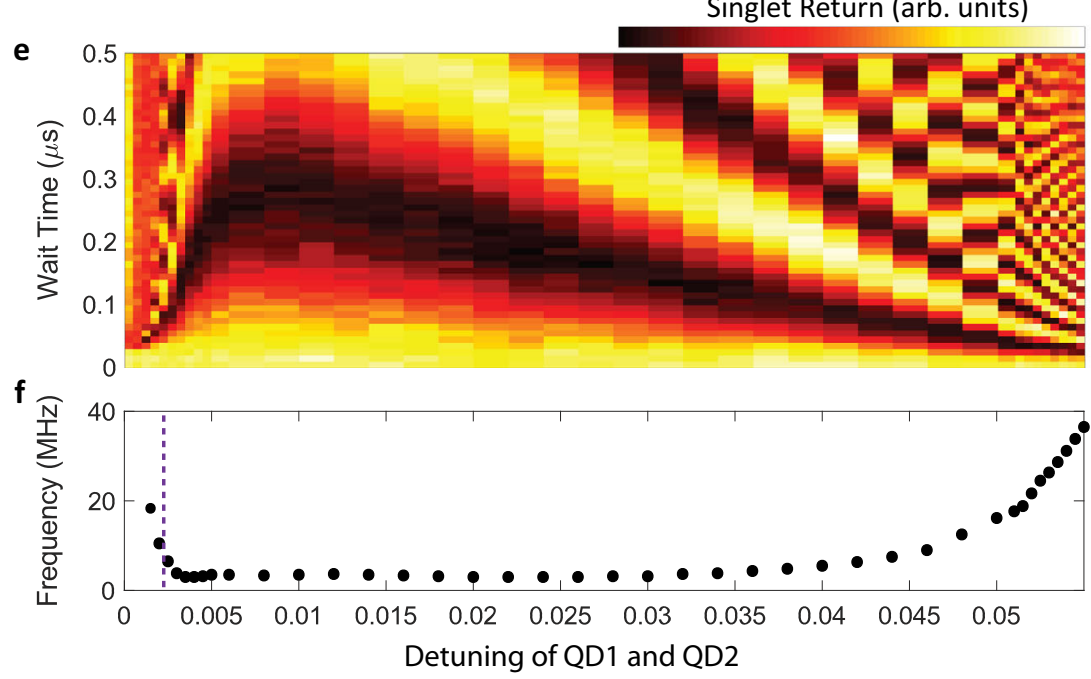

d

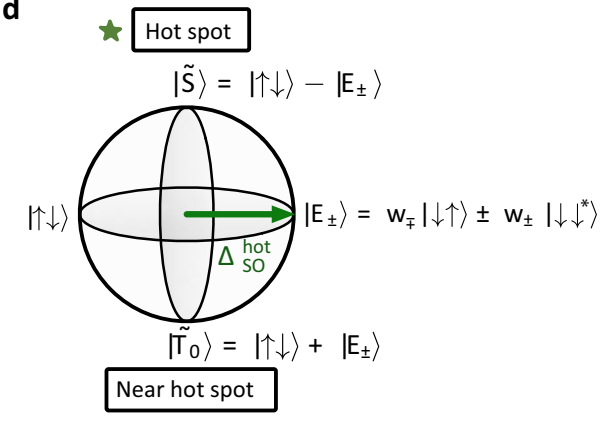

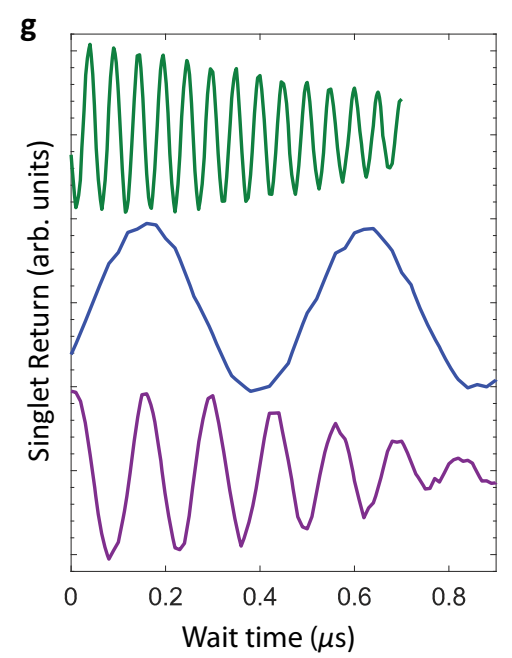

This has been corrected in both the PDF and HTML versions of the Article. 
The original version of this Article contained an error in Fig. 4, in which the font on the $\mathrm{x}$-axis in panel a was corrupted. The correct version of Fig. 4 is:
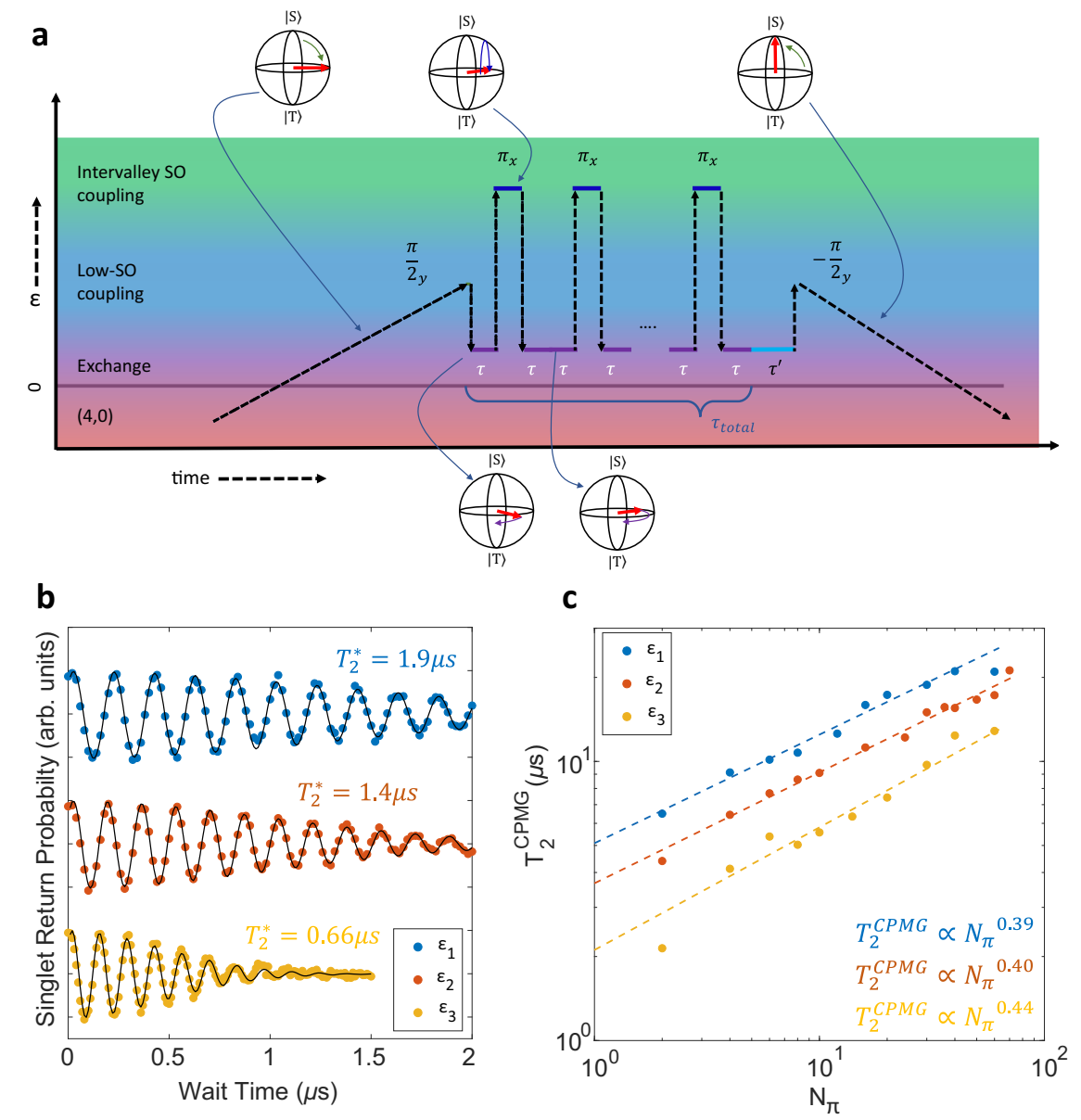
which replaces the previous incorrect version:
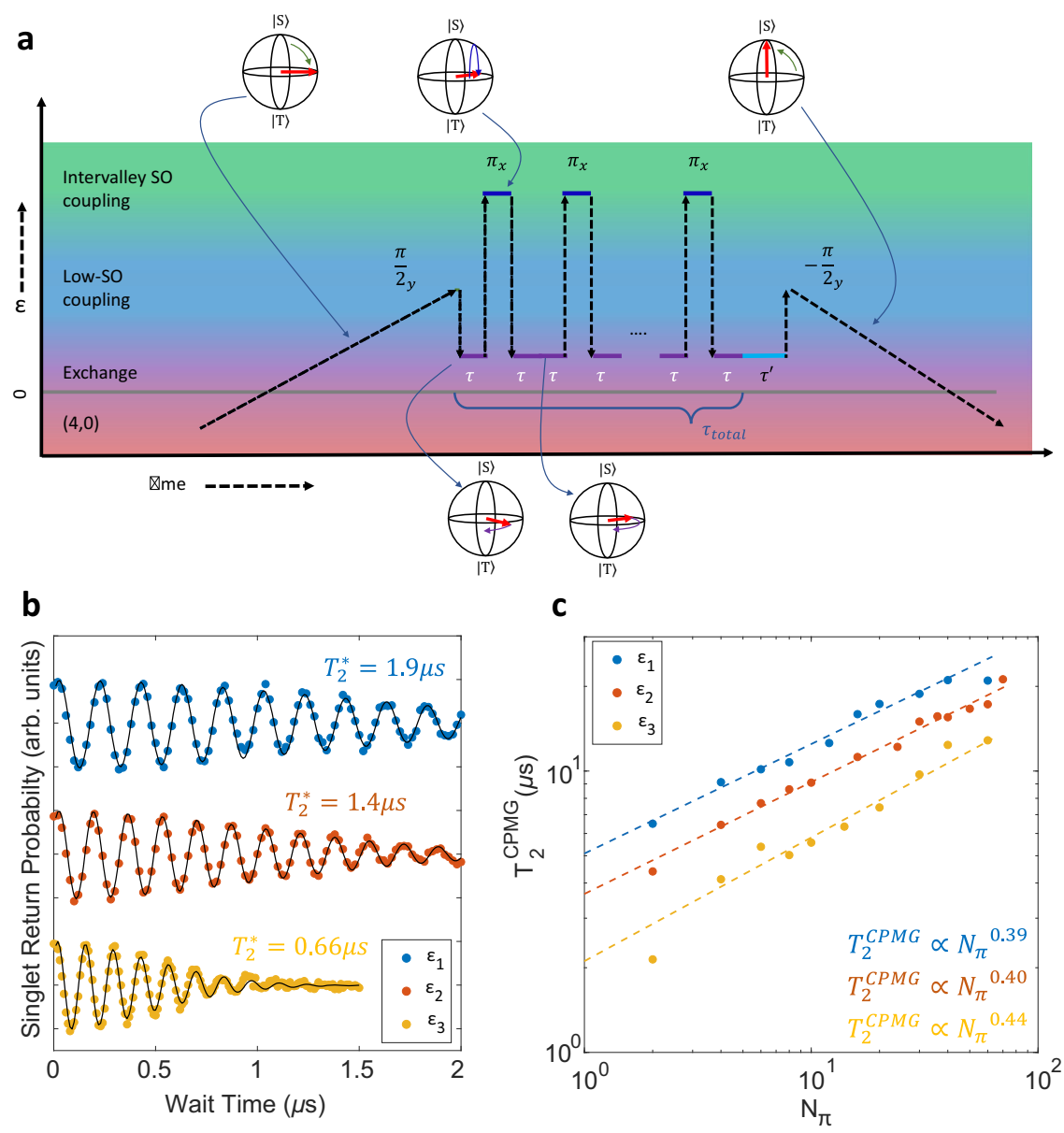

This has been corrected in both the PDF and HTML versions of the Article

In the original version of this Article, the copyright line was incorrectly given by "C) The Author(s) 2022". The correct copyright line is "() National Technology \& Engineering Solutions of Sandia, LLC 2022". This has now been corrected in the PDF and HTML versions of the Article.

Published online: 01 March 2022

\footnotetext{
(c) (i) Open Access This article is licensed under a Creative Commons Attribution 4.0 International License, which permits use, sharing, adaptation, distribution and reproduction in any medium or format, as long as you give appropriate credit to the original author(s) and the source, provide a link to the Creative Commons license,

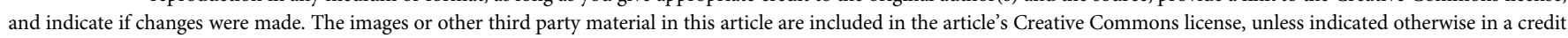
line to the material. If material is not included in the article's Creative Commons license and your intended use is not permitted by statutory regulation or exceeds the permitted use, you will need to obtain permission directly from the copyright holder. To view a copy of this license, visit http://creativecommons.org/licenses/by/4.0/.
} 